\title{
The phenotype strikes back and is out on more than one limb - A review of Developmental Plasticity and Evolution, by Mary Jane West-Eberhard
}

\author{
Klaus Hartfelder \\ Universidade de São Paulo, Faculdade de Medicina de Ribeirão Preto, Departamento de Biologia Celular, \\ Molecular e Bioagentes Patogênicos, Ribeirão Preto, SP, Brazil.
}

\begin{abstract}
Developmental Plasticity and Evolution. West-Eberhard M.J. (2003). Oxford University Press, New York. $x x+794$ pp. Hardcover 100 USD, paperback 49,95 USD. ISBN 0-19-512234-8; 0-19-512235-6 (pbk).
\end{abstract}

Received: November 12, 2004; March 18, 2005.

Writing a review on a book more than one year after its first publication may not seem like a good idea, but then, there are books that take their due time to be read. To biologists working on social insects, West-Eberhard is mainly known for her ground-breaking work and ideas on social evolution of wasps, so the theme of this current book may seem surprising. Yet, the caste systems of social insects are a first class cosmos of developmental plasticity and the role of the phenotype in evolution. Not surprisingly, therefore, the idea of major evolutionary change through alternative phenotypes has already sprung up in her earlier publications (for instance, West-Eberhard, 1989). The present book, however, is more than this, it is a milestone synthesis on the role of the environment in the shaping of evolutionary trajectories through their accommodation in developmental pathways. It is a strong argument for the primacy of the phenotype, and counters the currently still prevailing dominance of the genotype in evolutionary theory. Obviously, there is some risk to such an argument, namely that of falling short of a Darwinian point of view and succumbing to blunt Neo-Lamarckism. In the preface to her oeuvre, West-Eberhard clearly points out the arduous task to keep arguments straight and to steer clear of holistic muddle and new age vitalism. At the same time, it was necessary to keep in line with the rapidly expanding knowledge generated by evolutionary developmental biology. So, what was initially intended as a several page review turned into a 790 page book that took the author ten years to write, and, no wonder, a reader can and should spend due time on it.

Send correspondence to Klaus Hartfelder. Universidade de São Paulo, Faculdade de Medicina de Ribeirão Preto, Departamento de Biologia Celular, Molecular e Bioagentes Patogênicos, Avenida Bandeirantes 3900, 14040-900 Ribeirão Preto, SP, Brazil. E-mail: khartfel@rge.fmrp.usp.br.
The book is subdivided into four major sections, starting out with a Framework for a Synthesis. In the eight chapters dedicated to this section, West Eberhard presents a rare and critical review on the history of ideas on embryonic development and evolution, especially those that were formulated in parallel with the shaping of the "Modern Synthesis". The first chapter disentangles core concepts, some already shaped by Darwin, from their often times indiscriminate use. In particularly it focuses on concepts such as, adaptive peaks, epigenetic landscapes, developmental constraints. These concept are derived from a genotype-environment interaction point of view, and West-Eberhard now puts them under the perspective of condition-sensitive developmental processes that shape the phenotype. The second chapter, termed Material for a Synthesis, in a nutshell, already lays out the entire notion of the book and defines its major concepts for a synthesis of development and evolution, that is, how novelties can originate. It outlines the role(s) of heterochrony, canalization and constraints, reaction norms and polyphenisms and, what becomes an especially important line of the argument throughout the book, the concept of genetic assimilation and the Baldwin effect. The third chapter is an instructive introduction into developmental plasticity, yet a relatively standard one, especially for those who have already read Cells Embryos and Evolution (Gerhart and Kirschner, 1997). This chapter, also introduces one of the major examples on phenotypic accommodation, that makes its appearance at several points throughout the book. The example is a "two-legged goat" that, due to a birth defect, was born without forelegs and developed an upright posture and bipedal locomotion. Obviously, this example results from and represents adaptive mutual adjustments between parts of the body that turn it into a functional organismic continuity, without involving any genetic change. At the same time, this example illustrates the importance of developmental mechanisms that are organized as modules. And modularity in development is the theme of the next chapter.

Modularity in development does not only refer to metameric organization that characterizes the most advanced body plans of protostomes and deuterostomes, but also means modularity in regulatory networks, for instance 
Hox genes and signaling networks such as wingless, Notch/Delta and others. This means that modular elements can be employed sequentially and in different contexts of body plan organization and tissue differentiation. Consequently, and as West-Eberhard puts it (p. 87) "Modularity facilitates evolution both by use of already stable structural units and by the rapidity with which new complex structures can be assembled and quantitatively adjusted."

Chapters 5 (Development), 6 (Adaptive Evolution) and 7 (Principles of Development and Evolution) are core chapters to the entire book. First, they lay out the connection of developmental regulation by maternal and zygotic gene expression with environmental cues, by elaborating on the concept of ganged switches. In such switch mechanisms, endogenous and exogenous cues build upon each other and, once they reach threshold levels, they entrain developmental pathways. Under this point of view, genetic and environmental factors come to occupy levels of equivalence in impact on development and evolution, and this is one of the main (and also certainly one of the most controversial) points throughout the book. Conformingly, what can vary is the setting of threshold levels to cues in the entrainment of developmental pathways, and especially so, of alternative pathways leading to alternative phenotypes. Setting of threshold levels in the organization of switches, thus, becomes a point where selection enters as a player, and West-Eberhard emphasizes throughout all chapters that the unit of selection is the phenotype. So, the cues that have equal importance in ontogeny (genes and environment) also should be of equal importance in evolution. In fact, continuous or recurrent environmental change, through its effects on development, can be perceived as a very potent driving force in the shaping of the phenotype and the origins of novelty, and it is exactly in this context that West-Eberhard discusses how genetic accommodation, as a modified view of the Baldwin effect, could come into play. The fusion of development and evolution, which is a view already manifest in Darwin's theory (chapter 8 of this book) is a major theme in current developmental biology, and gains in comprehension when environmental effects are included as major agents of evolutionary change acting through development. This is the theme of chapter 7 where developmental plasticity and modularity, under the tutelage of environmental input, can, for instance, lead to character release and phenotypic recombination of characters. This chapter also raises the question of developmental plasticity in relation to genotype cohesiveness and speciation.

Section II, under the heading The Origins of Novelty comprises a series of 11 chapters that take up and provide illustrative examples on the concepts laid down in section I. Some of these chapters are outstanding in the compilation of informative biological examples that illustrate important and little understood points in development and evolution. Take for example chapter 15, entitled Cross-sexual trans$f e r$, which is a rare jewel on the link between alternative re- productive strategies and the sexually dimorphic phenotypes generated by endocrine switches that act on the background of a genetic or environmental system of sex determination. And in chapter 17, (Combinatorial evolution at the molecular level) West-Eberhard impressively demonstrates that she dominates not only the organismic level of evolutionary theory, but also the molecular basis and molecular prerequisites for the generation of evolutionary novelty. In this chapter, for example, she "somatizes" the information content of DNA by emphasizing its need for a functional nucleus-cytoplasm relationship. To a traditional gene concept this may seem a provocative point of view, but when taken in the context of current functional genomics, it actually knocks on open doors. The role of learning in phenotype organization is addressed in chapter 19 and juxtaposes the traditional view, according to which genetic change precedes behavioral change, to a view in which learning can take the lead, by matching a trait to conditions and, thus, driving behavioral phenotypes to genetic accommodation.

The question on how alternative phenotypes are generated and what is their potential in the evolution of novelties is introduced and discussed in Section III. Alternative phenotypes are generated by condition-sensitive switch mechanisms that trigger divergent differentiation programs during defined developmental phases. Condition sensitivity, that is sensitivity to environmental cues, does not mean that these alternatives are non-genetic. Rather, their genetic bases lies in the setting of the respective threshold values, and it is the threshold values that come under selection. This balance between environmental cues and a genetic background in the setting of thresholds generates distinct phenotypes that are adaptive under different environmental regimes, such as the diet-dependent morphs of tadpoles, the seasonal color and wing polyphenisms in butterflies, the density and season-dependent aphid polyphenisms, and, of course, the castes of social insects. Quite frequently, such alternative phenotypes have been classified by taxonomists as different species, which shows that quite striking novelties can be generated without speciation. Furthermore, already minor environmental change may tip the balance to one or the other alternative and lead to its fixation. A set of alternative phenotypes is, thus, par excellence material for potentially fast evolutionary change within lineages.

Section IV takes up the information and examples presented in Sections II and III and puts them in the context of the "big questions" outlined in Section I. Chapters 26 (Environmental Modifications), 27 (Speciation) and 28 (Adaptive Radiation) are highlights in this section. They outline how environmental influence can be reconciled with the genetic theory of adaptive evolution, by emphasizing genetic accommodation and interchangeability of genetic and environmental effects in developmental pathways. A developmentally plastic phenotype can respond to both genetic and environmental inputs, and the or- 
ganizational architecture of developmental switches can be a starting point for phenotypic evolution. As forcefully argued by West-Eberhard, a phenotypic novelty triggered by an environmental trend can arise simultaneously in many genotypes, and thus, should become established faster than one that was triggered by mutational change which, by definition, always starts in a single genotype. Under this perspective, speciation will not be the only or major driving force for divergence in evolutionary lineages, but only one of the possible consequences of phenotype divergence. The other one is the establishment of alternative phenotypes, that is, phenotypic divergence without speciation.

In this section, three textbook classics for adaptive radiation and speciation, the Hawaiian drosophilids, the Galapagos finches and African lake cichlids are thoroughly discussed under the notion of a developmentally flexible stem group. Flexible stem groups should rapidly respond to behavioral change induced by environmental possibilities, and they should allow to accommodate these genetically. In the case of the Hawaiian drosophilids, for example, the choice of new oviposition sites, and consequently new habitats for larval development, is accompanied by modifications in egg morphology and specializations in the female reproductive tract. On the larval side, the radiation is manifest in genetic changes at the alcohol dehydrogenase locus, which is a major effect locus for a larva's viability in and utilization of decaying fruit. The chapter closes with a new look on current evolutionary theory that puts away with the primacy of genotypic change:

"Genetic variation produces a response to selection, but it is patterned developmental plasticity that, by producing patterned variation, focuses selection in particular directions, and determines which genes are exposed to selection. This is why data on developmental plasticity are crucial to understand adaptive radiation".

The grand finale of this section are the chapters on Macroevolution (29) and Punctuation (30). The question of macroevolution is an issue that is rather evasively treated in Evolutionary Biology textbooks, mainly because it is not easily connected to classical population genetics. In contrast, the contextual framework of developmental biology easily ties major evolutionary change to changes in the temporal and spatial expression pattern of conserved key transcription factors and to signaling pathways. Developmental biology, in its evo-devo form, however could not - yet - provide a heuristic mathematical model which might rival the attraction of the population genetics-based paradigm of microevolution.

Is macroevolution then best be taken as a gradualistic extrapolation of microevolutionary steps, the gradualism view? Certainly not, since under this concept, macroevolution always means genotype-phenotype divergence above species level, whereas microevolution is primarily an issue of intraspecific, populational differentiation. West-Eberhard elegantly connects this apparent paradox to alternative phenotypes, which represent major phenotypic change within a species. And this, again, connects macroevolution to developmental plasticity, that is, major phenotypic change can occur without the need to appeal to macromutation hypotheses. It is in this perspective that West-Eberhard reiterates the statement of Eldredge and Gould (1972) when they proposed the speciational punctuated equilibrium hypothesis that "the expectations of theory color perception to such a degree that new notions seldom arise from facts collected under the influence of old pictures of the world", and she concludes with the bold statement that the plasticity hypothesis proclaimed in the present book "is the only evolutionary hypothesis that proposes an explicit and testable mechanism (changes in degree of plasticity or developmental versatility) as an alternative to speciation to explain both morphological stasis and punctuated change in terms of natural selection".

The line of arguments and especially the richness of examples to illustrate their key points are persuasive. But are we willing to follow her in these arguments and, especially, are they conclusive? Earlier reviewers of Developmental Plasticity and Evolution are quite divided on this question (de Jong and Crozier, 2003, Levinton, 2003; Rollo, 2004). They are unanimous on and underscore the profound knowledge of the author on biological facts and her ability to light-footedly but firmly transit through all levels of biological organization, and they also admit the possibility that current evolutionary theory may indeed be overemphasizing the central role of the genotype over the phenotype. Yet, they are divided on what are the implications of this hypothesis and where it may lead us to. The main challenge in this hypothesis clearly lies in the role attributed to the environment in evolutionary change, which is shifted from a force in the process of selection acting on mutational genotypic change to a force that actually may shape the genotype through accommodation on the phenotype side.

Clearly, even a book as brilliantly and convincingly written as the present one will hardly come to terms with such a century question, but it deserves full credit for having brought the phenotype back into the center of attention and to have firmly posed the problem of rapid phenotypic change in the context of developmental evolutionary biology. What is still lacking - and this has already been pointed out in the earlier reviews mentioned above - is the transition from a descriptive hypothesis on developmental plasticity and its role in evolution to a mathematically founded theory, which would turn it much more powerful and comparable to the classical genotype-oriented view. Rollo (2004) has illustrated this exquisitely in the title of his review "Life = epigenetics, ecology, and evolution $\left(L=E^{3}\right)$ ". An important step towards such an integration may, once again, come from evolutionary developmental biology, especially from the equally brilliant and authoritative book by Eric $\mathrm{H}$. Davidson, Genomic Regulatory Systems - Development 
and Evolution (2001) which outlines the power of promotor and upstream control region structure in the temporal and spatial organization of gene expression in development, and depicts what may be the consequences of rearrangements in such regulatory modules. Current attempts to model and simulate such changes in genomic regulatory networks (Bolouri and Davidson, 2003) may represent entry points into a mathematically founded theory for developmental plasticity and the role of the environment in evolutionary change.

One final word, tough books are hard to sell? It does not look like this since Developmental Plasticity and Evolution has within very short time found its way into the curricula of courses on evolutionary biology. Due to its provocative and refreshing ideas it challenges current mainstream (genotype-centered) evolutionary theory, without defecting Darwinian positions.

\section{References}

Bolouri H and Davidson EH (2003) Transcriptional regulatory cascades in development: initial rates not steady state, deter- mine network kinetics. Proc Natl Acad Sci, USA 100:9371-9376.

Davidson EH (2001) Genomic Regulatory Systems, Development and Evolution. Academic Press, San Diego, xii +261 pp.

De Jong J and Crozier RH (2003) A flexible theory of evolution. Nature 424:16-17.

Eldredge N and Gould SJ (1972) Punctuated equilibria: an alternative to phyletic gradualism. In: Schopf TJM (ed) Models in Paleobiology. Freemann, Cooper \& Co., San Francisco, pp 82-115.

Gerhart J and Kirschner M (1997) Cells, Embryos and Evolution. Blackwell Science, Malden, Massachusetts, xiii +642 pp.

Levinton JS (2003) Evolution as frozen music. Science 301:767-768.

Rollo CD (2004) Life = epigenetics, ecology, and evolution $\left(\mathrm{L}=\mathrm{E}^{3}\right)$ : A review of Developmental Plasticity and Evolution, by Mary Jane West-Eberhard. Evol Dev 6:58-62.

West-Eberhard MJ (1989) Phenotypic plasticity and the origins of diversity. Annu Rev Ecol Syst 20:249-278.

Associate Editor: André Luiz Paranhos Perondini 